\title{
INFINITY-NORM ROTATION FOR REVERSIBLE DATA HIDING
}

\author{
Lei Yang ${ }^{a} \quad$ Pengwei Hao ${ }^{a, b}$ \\ ${ }^{a}$ State Key Laboratory of Machine Perception, Peking University, Beijing 100871, China \\ ${ }^{\mathrm{b}}$ Department of Computer Science, Queen Mary University of London, London E1 4NS, UK \\ E-mail: \{yanglei, phao\}@cis.pku.edu.cn,phao@dcs.qmul.ac.uk
}

\begin{abstract}
In this paper, we propose a novel transform that preserves the dynamic range - infinity-norm rotation. This transform is perfectly reversible and piecewise linear, and keeps the maximum value unchanged. We apply the transform to reversible data hiding, which can be utilized for fragile data hiding and covert communication. After the inverse transform applied to the image with hidden data, no overflow or underflow occurs to the pixel values, and small changes of the coefficients in the transform domain result in small changes of the corresponding pixel values after reconstruction. With progressive symmetrical histogram expansion, we obtain very high embedding capacity for data hiding in the transform domain of infinity-norm rotation. Furthermore, the embedding capacity can be further expanded with blocked coefficients and low-frequency coefficients, which is demonstrated by our experiments.

Index Terms-Dynamic range preservation, histogram expansion, infinity-norm rotation, pyramidal hierarchy, reversible data hiding.
\end{abstract}

\section{INTRODUCTION}

Reversible data hiding, often referred to as reversible watermarking, was proposed as a promising technique for covert communication and information hiding. Such an embedding algorithm allows extraction of intact hidden data from the watermarked digital carriers and lossless recovery of the original images, when there is no malicious attack.

In the literature of reversible data hiding, the techniques can be mainly classified into two categories by different embedding domains. One type of reversible data hiding technique embeds data directly into the spatial domain. The representatives of this type are compressing general least significant bits (GLSB) to excavate space as embedding locations [1], and shifting histogram to empty certain bins for the data to be embedded [2]. The other type operates embedding in the transform domains [3-5]. These popular transforms, such as integer wavelet transform (IWT) and integer discrete cosine transform (IDCT), serve for compacting the coefficient distribution, and then facilitate the corresponding embedding rule to modify the centralized

\footnotetext{
* Supported by FANEDD(200038)\&NKBRPC(2004CB318005)of China.
}

coefficients. A linear transform with unitary determinant is also proved to be integer reversible if a PLUS factorization is applied to the transform matrix [8]. However, due to the dynamic range expansion problem [6] of these reversible transforms, embedding locations need to be carefully selected to avoid overflow and underflow of pixel values, which cuts down embedding capacity and complicates the algorithms. Therefore, the ideal transform for reversible data hiding should be perfect reversible, coefficient distribution compacted and dynamic range preserved. Besides, linearity or piecewise linearity of a transform is also desirable to guarantee that little change of coefficients results in little degradation of image quality after reconstruction. Piecewise Linear Haar-like transform (PLHaar) is such a transform, proposed by Senecal [7] for lossless and lossy coding, which is a special case of our proposed infinity-norm rotation.

In this paper, we propose a reversible transform that preserves the dynamic range-infinity-norm rotation. It possesses six interesting properties: (1) perfect reversibility; (2) dynamic range preservation; (3) piecewise linearity; (4) simple and fast implementation with in-place calculation; (5) coefficient distribution compaction; (6) separable and nonseparable implementation for arbitrary dimensional rotation. We will present the definition of infinity-norm rotation and a typical illustration of infinity-norm rotation in threedimensional space in Section 2. Furthermore, a fourdimensional infinity-norm rotation is used as a nonseparable transform with pyramidal hierarchy for reversible data hiding. The detailed embedding process using symmetrical histogram expansion is described in Section 3. When low-frequency coefficients are left unchanged, embedding into the blocked coefficients can further expand the embedding capacity even larger than $1 \mathrm{bpp}$. These results are given in Section 4, and in Section 5 come the conclusions.

\section{INFINITY-NORM ROTATION}

The ordinary rotation defined in Euclidean space, which is referred to as 2-norm rotation, is actually a rotation along a circle centered at the origin in $2 \mathrm{D}$ or on the surface of a sphere. The 2-norm of a point is its Euclidean length between the point and the origin. After 2-norm rotation, the length or the 2-norm of the point is preserved. Similarly, we 
can define $p$-norm rotations as that preserve $p$-norm after the rotations. For example, a transform that preserves 1-norm is a 1-norm rotation; a transform that keeps the infinity-norm unchanged is an infinity-norm rotation. Three rotations in $2 \mathrm{D}$ are illustrated in Fig.1, which shows the iso-distance curves of 1-norm, 2-norm and the infinity-norm. And the definition of $p$-norm rotation in higher dimensions is given below.

Definition 1. p-norm rotation

p-norm rotation that preserves p-norm, geometrically, is a move on the curve of the same p-norm.
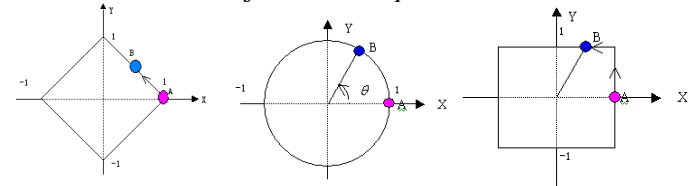

(a) 1-norm rotation (b) 2-norm rotation (c) infinity-norm rotation

Fig. 1 rotations in 2D

In two-dimensional space, infinity-norm rotation is a move along a square (shown in Fig.1(c)); in threedimensional space, an infinity-norm rotation is a move on the surface of a cube (shown in Fig.4); in higher dimensional space, infinity-norm rotation is a move on the surface of a hyper cube.

Definition 2. Angle of p-norm rotation

Angle of p-norm rotation is the ratio of the runlength between two points along an equal p-norm curve to the radius of the curve.

$$
\theta_{p}=\frac{\text { runlength }}{\text { radius }}
$$

where the radius is defined as the p-norm of the curve.

Especially, $\theta_{\infty}=$ runlength $/($ sidelength $/ 2)$, when rotating along a square in 2D; $\theta_{2}=$ arclength/radius is the angle measure of a circular rotation. An approximated relation between $\theta_{\infty}$ and $\theta_{2}\left(\theta_{2} \in[0, \pi / 4]\right)$ can be defined as $\theta_{\infty}=\operatorname{tg} \theta_{2}$, $\theta_{\infty}=2$ when $\theta_{2}=\pi / 2$. Other relations can be found by combining the above rotations. An infinity-norm rotation from point $\mathrm{A}$ to point $\mathrm{B}$ by an angle $\theta_{\infty}$ is denoted as $B=R_{\infty}\left(A, \theta_{\infty}\right)$ in following discussions.

\section{Properties of infinity-norm rotation:}

1) Linearity:

$$
X \in \Re^{2}, R_{\infty}\left(X, a \cdot \theta_{\infty}+b \cdot \theta_{\infty}\right)=a \cdot R\left(X, \theta_{\infty}\right)+b \cdot R\left(X, \theta_{\infty}\right)
$$

2) Infinity-norm preservation:

3) Periodicity:

$$
\|X\|_{\infty}=\left\|R_{\infty}\left(X, \theta_{\infty}\right)\right\|_{\infty}
$$

$$
X \in \Re^{2}, R_{\infty}\left(X, \theta_{\infty}\right)=R_{\infty}\left(X, \theta_{\infty}+8\right)
$$

The period of the infinity-norm rotation is 8 , while the 2 norm rotation has a period of $2 \pi$.

4) Bijection:

$X \in \mathfrak{R}^{2}, \alpha_{\infty}, \beta_{\infty} \in[0,8), R_{\infty}\left(X, \alpha_{\infty}\right)=R_{\infty}\left(X, \beta_{\infty}\right) \Leftrightarrow \alpha_{\infty}=\beta_{\infty}(5)$

5) Piecewise linear transforms:

An infinity-norm rotation can be represented by piecewise linear transforms in Euclidean space. In 2D, as shown in Fig.2, we can divide the space into 8 regions. In each region, the infinity-norm rotation is a linear transform which is a shear transform or a product of reflection and shear transform.

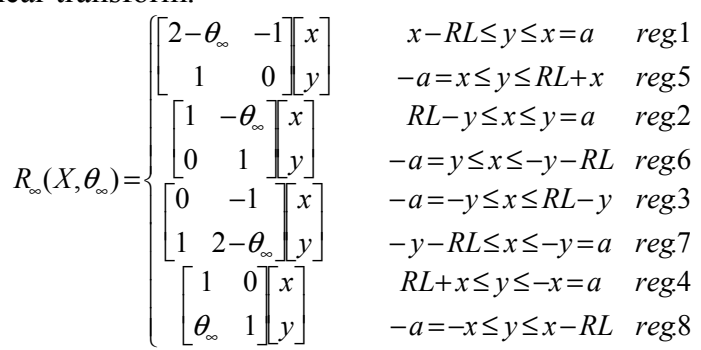

where $X=(x, y), a=\|X\|_{\infty}$ and $R L=a \cdot \theta_{\infty}$.

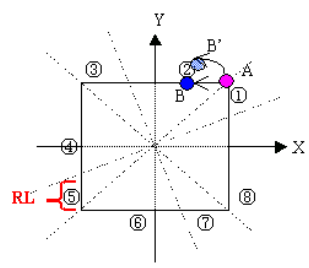

Fig.2 piecewise linearity of infinity-norm rotation

In discrete space, if the dynamic range of data is the integers from 0 to 255 , the domain and the range of $n$ dimensional infinity-norm rotation are both a $\underbrace{256 \times 256 \times \cdots \times 256}_{n}$ lattice constructed by the sub-lattices whose nodes have the same infinity-norm. The runlength for each sub-lattice should also be discretized. When $X=(x, y), a=\|X\|_{\infty}$,

$$
\text { runlength }=\left\lfloor a \cdot \theta_{\infty}\right\rfloor
$$

where $\lfloor\cdot\rfloor$ is a rounding operator which can be round, ceil or floor, to constrain runlength to have the same precision as the nodes on the lattice. Except runlength, only addition and subtraction are needed during the in-place calculation of infinity-norm rotation. It is feasible for hardware implementation with limited buffer, cheap fixed-point arithmetic unit and fixed-width channels.

To find the proper rotation angles, we use the tool of $n D$ co-histogram of an image for analysis.

\section{Definition 3. $n D$ co-histogram}

$n D$ co-histogram is an n-dimensional histogram for the statistics of the co-occurrence of $n$-dimensional points whose coordinates are the values of every $n$ neighbor pixels in the image (see [9] for 2D co-histogram).

The angles of infinity-norm rotation are chosen such that the diagonals in Fig. 3(a)(d) parallel to an axis. After rotation, the dimension parallel to an axis is mostly kept invariant, which represents the low-frequency components of the images, while the other dimensions are high-frequency components of the images. The two-dimensional infinitynorm rotation as shown in Fig.1(c) results in Fig.3(c). 
Fig.3(b) is for 2D DCT as a 2-norm rotation for comparison. In three-dimensional discrete space, infinity-norm rotation, denoted as $R_{\infty}^{3}$, takes place on the surface of a cube as illustrated in Fig.4. $R_{\infty}^{3}$ is the rotation around the center of the cube, and turns the diagonal of the cube parallel to an axis. The points of the similar colors are on a rectangle, which rotate counter-clockwise along the rectangle. The ones in light color disappearing from one face will appear in another face. Similarly, it can be generalized into arbitrary dimensional infinity-norm rotation. After $R_{\infty}^{3}$ is applied to image Lena, the result is shown in Fig.3(f), in comparison to the result after DCT, a 2-norm rotation, shown in Fig.3(e).

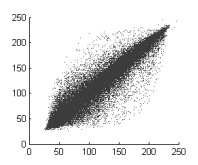

(a) $2 \mathrm{D}$ original

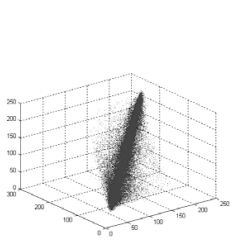

(d) 3D original

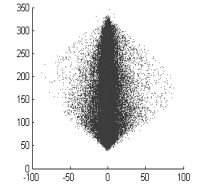

(b) 2D DCT

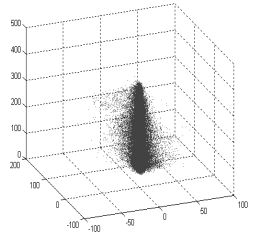

(e) 3D DCT

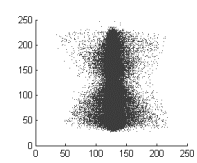

(c) $R_{\infty}^{2}$

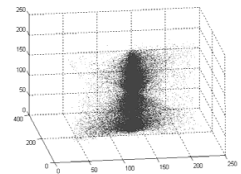

(f) $R_{\infty}^{3}$
Fig.3 co-histograms of Lena

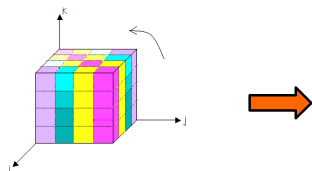

(a) before $R_{\infty}^{3}$

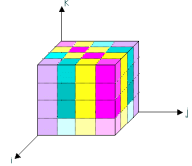

(b) after $R^{3}$
Fig. 4 the three-dimensional data on the surface of a cube

After infinity-norm rotation around the middle pixel values 127 and 128, the coordinates of the points are centralized to 127 and 128. The overall histogram of coefficients is shown in Fig. 5(c), which is suitable for reversible data hiding using symmetrical histogram expansion.

For four-dimensional infinity-norm rotation, a nonseparable transform with pyramidal hierarchy is illustrated in Fig.5(a) to show multiresolusion analysis of $R_{\infty}$ to images.

The 2-by-2 blocks in the image are taken as the fourdimensional points to be rotated because they have higher correlations than 1-by-4 or 4-by-1 blocks. The yellow arrows in Fig.5(a) show the scan order inside the blocks.

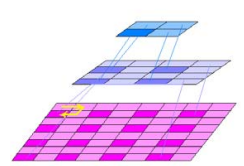

(a) pyramidal hierarchy

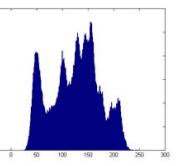

(b) before $R_{\infty}^{4}$

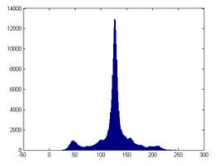

(c) after $R_{\infty}^{4}$
Fig.5 non-separable $R_{\infty}^{4}$ and the histograms of Lena

\section{REVERSIBLE DATA HIDING}

\subsection{Symmetrical histogram expansion}

For the convenience of description, the $n-b u d d y$ definition is presented first.

Definition 4. $n-b u d d y$

An $n$-buddy of an m-bit number is an m-bit number, whose upper $m-n$ bits are the same, but the lower $n$ bits have at least one bit different.

Take 8-bit numbers as examples: 2 and 3 are 1-buddies of each other, and 4, 6, 7 are all 2-buddies of 5. It is obvious that if a number is modified in the lower $\mathrm{n}$ bits, it must be one of its n-buddies or itself. Therefore, after the lower $n$ bits of the coefficients in a histogram bin are modified, the pixel value in the bin expands into the empty neighbor nbuddy bins. In the recovery process, these neighbor n-buddy bins are all mapped into the original single bin. The lower $n$ bit modification scheme for embedding process can be formulated as:

$$
C_{w}=\left\lfloor C / 2^{n}\right\rfloor \cdot 2^{n}+W
$$

Fig. 6 illustrates our symmetrical histogram expansion scheme. Firstly, we choose bin 6 as the pivotal bin for the first embedding and shift bin 7 to the right as bin 8 to evacuate bin 7 for the embedding. Then we shift bin 4 to the left to embed data into bin 5. Finally, we embed data into bin 8 . That is for data embedding in a zigzag scanning order. For hidden data extraction, we convert pixel values 6 and 7 into 6,5 and 4 into 5,3 into 4,8 and 9 into 7 so as to recover the original coefficient values in the same order as in embedding.
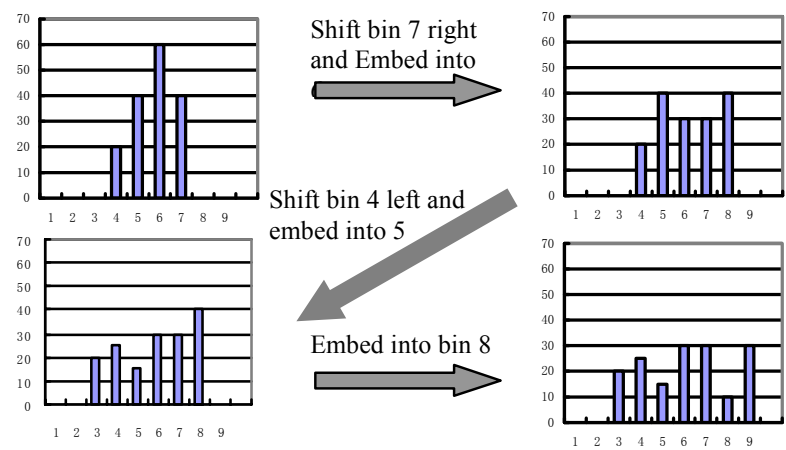

Fig.6 symmetrical histogram expansion for data embedding

\subsection{Data embedding and extraction}

Based on the four-dimensional infinity-norm rotation and the symmetrical histogram expansion scheme, our progressive algorithm is summarized as follows.

Data embedding:

1. Transform the original image using $R_{\infty}^{4}$.

2. Attach the lower bits of the coefficients in the first row to the data to be embedded. 
3. Choose 128th bin in the coefficient histogram as the pivot, and record the length of the data to be embedded into the lower bits of the coefficients in the first row.

4. While there are data left to be embedded do

Expand the histogram symmetrically;

Modify the lower $n$ bits of the coefficients.

\section{End while}

5. Apply the inverse $R_{\infty}^{4}$ and output the image with data.

Data extraction and image recovery:

1. Transform the image with hidden data using $R_{\infty}^{4}$.

2. Extract the pivot value and the length of the hidden data from the coefficients in the first row.

3. While there are data left to be extracted do Extract the lower $n$ bits of the coefficients; Shrink the expanded histogram back.

\section{End while}

4. Recover the coefficients in the first row.

5. Apply the inverse $R_{\infty}^{4}$ to recover the original image.

In our algorithm, we not only shift one bin for 1-bit modification, but also shift three and seven bins for 2-bit and 3-bit modification to increase the capacity.

\section{EXPERIMENTAL RESULTS}

Using pyramidal four-dimensional rotation, we list the capacity (bytes) in each second row and PSNR (dB) in each first row for the test images with different analysis levels. Three analysis levels are optimal for symmetrical histogram expansion in our experiments. Partition the coefficients into blocks in the same size as that of low-frequency block at the highest analysis level. Then leave the incompact lowfrequency coefficients unchanged. The histogram of each high-frequency block has the distribution like Fig. 5(c), but more compact and more empty bins at both sides. We use three level analyses, divide the coefficients into $8 * 8$ blocks, and show the results as $3^{*}$ in the sixth column of Table 1 . The embedding capacity of some images exceeds $1 \mathrm{bpp}$, and can still be further expanded, if image quality does not matter much.

Table 1 Capacity (bytes) and PSNR (dB) for the test images

\begin{tabular}{|c|c|c|c|c|c|}
\hline Image & 1 & 2 & 3 & 4 & $3^{*}$ \\
\hline \multirow{2}{*}{ Barbara } & 31.34 & 31.84 & 32.11 & 30.77 & 24.24 \\
\cline { 2 - 6 } & 15912 & 19027 & 24001 & 24098 & 29315 \\
\hline \multirow{2}{*}{ Lena } & 31.92 & 32.77 & 32.93 & 31.49 & 24.53 \\
\cline { 2 - 6 } & 19866 & 23683 & 26716 & 26802 & 37491 \\
\hline \multirow{2}{*}{ Boat } & 31.67 & 32.16 & 32.37 & 30.96 & 24.02 \\
\cline { 2 - 6 } & 18266 & 22327 & 27674 & 27700 & 37949 \\
\hline \multirow{2}{*}{ Jet } & 31.87 & 32.68 & 32.73 & 31.03 & 23.68 \\
\cline { 2 - 6 } & 19743 & 24153 & 28629 & 28631 & 42433 \\
\hline \multirow{2}{*}{ Baboon } & 31.38 & 31.22 & 30.15 & 29.26 & 23.94 \\
\cline { 2 - 6 } & 10732 & 12286 & 13192 & 13296 & 15986 \\
\hline \multirow{2}{*}{ Gold } & 31.65 & 32.27 & 32.30 & 31.09 & 24.33 \\
\cline { 2 - 6 } & 16457 & 19484 & 22618 & 22748 & 28294 \\
\hline
\end{tabular}

As in Table 2, we compare the performance of our proposed method with the good performance of the reversible data hiding methods in the recent literature on Lena and highly textured Baboon. It is obvious that our method have good average performance at high embedding bit rate.

Table 2 Capacity (in bytes) and PSNR (dB) comparison between reversible data hiding methods at high bit rates

\begin{tabular}{|c|c|c|c|c|}
\hline \multirow{2}{*}{ Method } & \multicolumn{2}{|c|}{ Lena $(512 * 512 * 8)$} & \multicolumn{2}{c|}{ Baboon $(512 * 512 * 8)$} \\
\cline { 2 - 5 } & Capacity & PSNR & Capacity & PSNR \\
\hline Tian [3] & 27755 & 32.54 & N/A & N/A \\
\hline Xuan et al [4] & 19661 & 34.39 & 13107 & 30.69 \\
\hline Celik et al [1] & 22127 & 31.9 & 5793 & 31.9 \\
\hline Ni et al [2] & 683 & 48.2 & 678 & 48.2 \\
\hline Proposed & 26716 & 32.93 & 13191 & 30.15 \\
\hline
\end{tabular}

\section{CONCLUSIONS}

In this paper, we propose a piecewise linear transform that preserves the dynamic range - infinity-norm rotation, and apply it to reversible data hiding. The embedding capacity is very high. Furthermore, infinity-norm rotation can be generalized into arbitrary dimensional transform and have diversified combinations. Which should be effective for reversible data hiding merits further investigation.

\section{REFERENCES}

[1] M. Celik, G. Sharma, A. Tekalp and E. Saber, "Lossless generalized-LSB data embedding," IEEE trans. on image processing, Vol.14, No.2, February 2005.

[2] Z. Ni, Y. Shi, N. Ansari and W. Su, "Reversible data hiding," IEEE Trans. on Circuits and Systems for Video Technology, Vol.16, No.3, March 2006.

[3] J. Tian, "Reversible data embedding using a difference expansion," IEEE Trans. on Circuits and Systems for video Technology, Vol.13, No.8, August 2003.

[4] G. Xuan, J. Zhu, J. Chen, Y. Shi, Z. Ni and W. Su, "Reversible data hiding on wavelet spread spectrum," IEEE Proceedings of Multimedia Signal Processing Workshop, Siena, Italy, Sept. 2004.

[5] B. Yang, M. Schmucker, W. Funk, C. Busch and S. Sun, "Integer DCT-based reversible watermarking for images using companding technique," Proceeding of SPIE, Security and watermarking of Multimedia Content, San Jose (USA), 2004.

[6] A. Kiely and M. Klimesh. "The ICER progressive wavelet image compressor," The Interplanetary Network Progress Report 42 155, July-September 2003, Jet Propulsion Laboratory, pp. 1-46, Nov 2003.

[7] J. Senecal, M. Duchaineau and K. Joy, "An improved n-bit to n-bit reversible Haar-like transforms," Pacific Conference on Computer Graphics and Applications, August 16-18, 2004.

[8] P. Hao, "Customizable triangular factorizations of matrices," Linear Algebra Appl., pp. 135-154, 282, 2004.

[9] P. Hao, C. Zhang, A. Dang, "Co-histogram and image degradation evaluation," IEEE Conf. ICIAR, pp. 195-203, 2004. 